\title{
ON $N^{x_{1}}$ AND THE ALMOST-LINDELÖF PROPERTY
}

\author{
STEPHEN H. HECHLER 1
}

ABSTRACT. In 1970, Kemperman and Maharam proved that there exists a Baire measure $\mu$ on $N^{c}$ (where $N$ is the set of natural numbers) such that $N^{\mathbf{c}}$ may be covered by a famliy of elementary open $\mu$-null sets and used this to prove that $R^{c}$ (where $R$ is the set of real numbers) does not have the "almost-Lindelöf" property. We define $K$ to be the smallest cardinal $\kappa$ for which there exists a collection of $\kappa$ closed subsets of $R$ each of Lebesgue measure zero and which covers $R$, and we show that in the above results $c$ can be replaced by $K$. We then note that we have shown elsewhere that it is consistent with the negation of the continuum hypothesis that $K=\aleph_{1}$, and this, therefore, implies that it is consistent with the negation of the continuum hypothesis that $R^{\aleph_{1}}$ not be almost-Lindelöf.

As in [4], a topological space $T$ is defined to be almost Lindelöf iff for every Baire measure $\mu$ on $T$ and every open cover $\mathcal{G}$ of $T$ there exists a countable family $\mathcal{H} \subseteq \mathcal{G}$ such that $T-\bigcup \mathcal{H}$ is $\mu$-null. In [4] J. H. B. Kemperman and Dorothy Maharam consider the question, due to H. Rubin, as to whether or not $R^{X_{1}}$ (where $R$ is the real line) is almost Lindelöf. They show that $R^{\mathbf{c}}$ is not almost Lindelöf thus settling the problem when given the continuum hypothesis, and, in fact, they prove a much stronger result. They do this by constructing a certain map from the set $I$ of irrationals between zero and one into the product $N^{c}$ (where $N$ is the set $\{1,2,3, \ldots\}$ of natural numbers), using this map to define a Baire measure and an appropriate cover on $N^{c}$, and then extending both to $R^{\mathbf{c}}$.

In this paper we shall define an uncountable cardinal $\mathbf{K} \leq \mathbf{c}$, and we shall show how the above construction can be modified to apply to $N^{\mathbf{K}}$ and, therefore, to $R^{\mathrm{K}}$. We shall then note that elsewhere [1] we have proven the consistency of $K=\boldsymbol{N}_{1}$ with the negation of the continuum hypothesis and thus prove that it is consistent with the negation of the continuum hypothesis that $R^{\boldsymbol{K}_{1}}$ not be almost Lindelöf.

We begin by letting $\lambda$ denote Lebesgue measure, and we define a family of closed subsets of $R$ to be a $\lambda-F$-cover iff it covers $R$ and each of its members has Lebesgue measure zero. Then let $\mathbf{K}$ be the smallest cardinal $\kappa$ for which there exists a $\lambda-F$-cover of cardinality $\kappa$. That $\mathbf{K}$ exists follows from the axiom of choice (which implies that every set of cardinals has a least

Received by the editors March 15, 1974 and, in revised form, July 4, 1974. AMS (MOS) subject classifications (1970). Primary 02K05, 54A25; Secondary 02K25, 04A30, 28A35.

1 The preparation of this paper was partially supported by a grant from the City University of New York Faculty Research Program, \#10621. 
member and which we shall assume without further mention); that it is at most $\mathbf{c}$, from the fact that the family of all singletons of $R$ forms a $\lambda_{-} F$-cover and that it is uncountable, from the fact that each member of the cover has Lebesgue measure zero. Now we modify the construction in [4] to prove

Theorem 1. There exists a function $\psi$ from $I$ into $N^{\mathrm{K}}$ and an elementary open cover $\mathcal{G}$ of $N^{\mathrm{K}}$ such that:

(a) If $U$ is any elementary open subset of $N^{\mathrm{K}}$, then $\psi^{-1}[U]$ is a Borel set in $I$.

(b) If $G$ is any member of $\zeta$, then $\lambda\left(\psi^{-1}[G]\right)=0$.

Proof. Let $\mathcal{C}^{*}$ be any $\lambda$-F-cover of cardinality $\mathbf{K}, \mathcal{C}$ the family $\{C \cap I$ : $C \in \mathcal{C}^{*}$ and $\left.C \cap I \neq \varnothing\right\}$, and since it is well known that $I$ is isomorphic to the product space $N^{N}$ (where $N$ has the discrete topology), identify $I$ with this space. Thus a member of $I$ will be an infinite sequence of members of $N$, and a member of $\mathcal{C}$ will be a set of such sequences. Also, it will be convenient to represent $N^{\mathrm{K}}$ as $N^{N} \times N^{\mathcal{C}}(R-I$ is countable, so $\mathcal{C}$ must have cardinality $\mathbf{K})$, and so a point in $N^{\mathrm{K}}$ will be a pair $(x, y)$ where $x=\left(x_{1}, x_{2}, \ldots\right)$ is a sequence of points in $N$ and $y$ is a function from $\mathcal{C}$ into $N$.

We now define our function $\psi: I \rightarrow N^{\mathrm{K}}$ by setting $\psi(x)=\left(x, y_{x}\right)$, where $y_{x}$ is the function from $\mathcal{C}$ into $N$ defined by

(i) $y_{x}(C)=1$ if $x \in C$,

(ii) $y_{x}(C)=\inf \left\{n \in N: r \notin C\right.$ whenever $r_{k}=x_{k}$ for $\left.1 \leq k<n\right\}$ if $x \notin C$. We note that in each instance of (ii) the value $y_{x}(C)$ is well defined and strictly greater than 1 because each $C \in \mathcal{C}$ is closed and nonempty.

To prove that $\psi^{-1}$ applied to elementary open sets yields Borel sets, it is sufficient to note that sets of the form $\left\{x \in N^{N}: x_{k}=n\right\}$ or $\left\{x \in N^{N}\right.$ : $\left.y_{x}(C)=n>1\right\}$ are open, while those of the form $\left\{x \in N^{N}: y_{x}(C)=1\right\}=C$ are closed. Thus $\psi^{-1}$ of an elementary open set is, at worst, both $\mathbf{F}_{\sigma}$ and $\mathbf{G}_{\delta}$.

Next we define $\mathcal{G}$. For each $r \in C \in \mathcal{C}$ and each $n \in N$ we set

$$
G_{n}(r, C)=\left\{(x, y) \in N^{\mathrm{K}}: y(C)=n \quad \text { and } \quad k<n \rightarrow x_{k}=r_{k}\right\}
$$

and we set

$$
乌=\left\{G_{n}(r, C): n \in N \text { and } \quad r \in C \in \mathcal{C}\right\}
$$

To see that $\mathcal{G}$ is a cover, choose any $(x, y) \in N^{\mathrm{K}}$. Then $x$ belongs to $I$ and, therefore, to some $C \in \mathcal{C}$. Thus $(x, y) \in G_{y(C)}(x, C)$.

Finally, we note that

$$
\psi^{-1}\left[G_{n}(r, C)\right]= \begin{cases}C & \text { if } n=1 \\ \varnothing & \text { otherwise }\end{cases}
$$

and that in either case we have $\lambda\left(\psi^{-1}\left[G_{n}(r, C)\right]\right)=0$. 
We note that if $\mathbf{K}=\mathbf{c}$ and we set $\mathcal{C}=\{\{r\}: r \in I\}$, the above construction reduces to that in [4].

While we do not know if can replace $\mathbf{K}$ by $\boldsymbol{\kappa}_{1}$ in Theorem 1 when $\mathbf{K}$ is strictly greater than $\kappa_{1}$, and we do not even have complete information as to all of the possible values of $\mathbf{K}$, it can be seen that the set $\left\{F_{a}: a<\kappa\right\}$ constructed in [1] is a $\lambda-F$-cover of cardinality $\kappa$, and, therefore, from the related results in [1] that it is consistent with the negation of the continuum hypothesis that $\mathbf{K}$ be any regular uncountable cardinal less than or equal to c. Thus we have

Theorem 2. It is consistent with the negation of the continuum bypothesis that $R^{\aleph_{1}}$ not be almost Lindelöf. In particular, it is consistent that there exists a Baire measure $\mu$ on $R^{\boldsymbol{N}_{1}}$ such that $\mu\left(R^{\boldsymbol{N}_{1}}\right)=1$ and that there exists an open cover of $R^{\aleph_{1}}$ by elementary $\mu$-null sets.

Proof. The proof is exactly as [4] except that we use our function $\psi$ and our cover $\mathcal{G}$ as obtained using Theorem 1.

We note that a closed set of measure zero must be nowhere dense, so if we let $\mathbf{K}_{N}$ denote the number of nowhere dense sets needed to cover $R$, and we let $K_{L}$ denote the number of sets of Lebesgue measure zero needed to cover $R$, then we have $\mathbf{K} \geq \max \left(\mathbf{K}_{N}, \mathbf{K}_{L}\right)$. It would be interesting to know if this inequality can ever be strict. It is known that given Martin's axiom [5] all three cardinals are equal to $\mathbf{c}$, and it is also known [6] that if "generic" reals are added, $\mathbf{K}_{N}$ becomes equal to $\mathbf{c}$ and $\mathbf{K}_{L}$ remains equal to $\boldsymbol{K}_{1}$, while if "random" reals are added, the reverse occurs. We also note that elsewhere [2], [3] we have studied a related cardinal, namely, the cardinality of the smallest compact cover of $I$.

We wish to thank the referee for his extremely useful suggestions concerning the notation in the proof of Theorem 1 .

\section{REFERENCES}

1. S. H. Hechler, Independence results concerning the number of nowhere dense sets necessary to cover the real line, Acta Math. Acad. Sci. Hungar. 24 (1973), 2732. MR $47 \# 1611$.

2. - Exponents of some N-compact spaces, Israel J. Math. 15 (1973), $384-395$.

3. - On a ubiquitous cardinal, Proc. Amer. Math. Soc. 52 (1975), 348-352.

4. J. H. B. Kemperman and D. Maharam, $R^{c}$ is not almost Lindelöf, Proc. Amer. Math. Soc. 24 (1970), 772-773. MR $40 \# 6500$.

5. D. A. Martin and R. M. Solovay, Internal Cohen extensions, Ann. Math. Logic 2 (1970), 143-178. MR 42 \#5787.

6. R. M. Solovay, A model of set theory in which every set of reals is Lebesgue measurable, Ann of Math. (2) 92 (1970), 1-56. MR 42 \#64.

DEPARTMENT OF MATHEMATICS, QUEENS COLLEGE OF THE CITY UNIVERSITY OF NEW YORK, FLUSHING, NEW YORK 11367 